\title{
Análises mecânicas e físico-químicas de couros de tilápia, cachara e salmão
}

\author{
Yoshida, G.M. ${ }^{1 @ ; ~ K u n i t a, ~ N . M . ~}{ }^{2 ;}$ Souza, M.L.R. ${ }^{2}$ e Gasparino, E. ${ }^{2}$
}

"Universidade Estadual Paulista "Júlio de Mesquita Filho". Faculdade de Ciências Agrárias e Veterinárias (UNESP - FCAV/Jaboticabal). Jaboticabal. Brasil.

${ }^{2}$ Universidade Estadual de Maringá. Maringá. Brasil.

PALAVRAS-CHAVE ADICIONAIS

Determinação da tração.

Determinaç̃õo do óxido de cromo.

Rasgamento progressivo.

Resistência dos couros.

\section{RESUMO}

Objetivou-se comparar as análises físico-químicas e mecânicas de couros de tilápia do Nilo, cachara e salmão submetidos à mesma técnica de processamento da pele. As peles de tilápias, salmão e cachara foram obtidas da unidade de beneficiamento Smart fish (RolândiaPR), Empresa Tomita e Tomita (Maringá-PR) e Embrapa (Corumbá-MS), respectivamente. Assim que retiradas dos peixes, foram submetidas ao congelamento para transporte e estocagem até ao momento de realizar o curtimento. As peles para o curtimento, foram descongeladas, descarnadas e submetidas à mesma técnica de processamento e transformadas em couro. $O$ couro de tilápia apresentou a maior espessura, força de carga de ruptura e de tração, o de salmão apresentou a maior resistência ao rasgamento $(105,69 \mathrm{~N} / \mathrm{mm})$. O couro de cachara apresentou resultados inferiores aos couros de tilápia e salmão quanto a porcentagem de elongação. O couro de salmão apresentou o melhor percentual de óxido de cromo $(2,6 \%)$, já para a tilápia $(2,0 \%)$ e para a cachara $(1,8 \%)$, os valores ficaram abaixo do recomendando para couros curtidos com sais de cromo. Substâncias extraíveis em diclorometano para salmão foi o mais indicado $(14,7 \%)$, enquanto os demais ficaram acima do valor recomendado, sendo necessário ajustes na formulação para a obtenção de couros de melhor qualidade.

\section{Mechanical and physical-chemical analysis of tilapia, cachara and salmon leather}

\section{AdDITIONAL KEYWORDS}

Determination of traction.

Determination of chromium oxide.

Progressive tearing.

Resistance of leather.

\section{INFORMAÇAO}

\section{Cronología del artículo.}

Recibido/Received: 15.01 .2016

Aceptado/Accepted: 06.05.2016

On-line: 15.09 .2016

Correspondencia a los autores/Contact e-mail:

grazyoshida@hotmail.com

\section{SUMMARY}

The objective was to compare the physicochemical and mechanical analysis of the Nile tilapia, cachara and salmon leather under the same skin processing technique. The tilapia, salmão and cachara skins were obtained from the processing unit Smart fish (Rolândia-PR), Tomita e Tomita (Maringá-PR) and Embrapa (Corumbá-MS), respectively. After removing the skins from the fish, they were subjected to freezing for their transport and storage until the time of performing the tanning process. The hides for tanning were thawed, stripped and subjected to the same processing technique and processed into leather. The leather tilapia showed the greatest thickness, tensile strength, and tensile strength, while the salmon had the highest tear resistance $(105.69 \mathrm{~N} / \mathrm{mm})$. Cachara leather results were lower than those of the leather of tilapia and salmon concerning their elongation percentage. Salmon leather showed the best chromium oxide percentage $(2.6 \%)$, while this was $2.0 \%$ for tilapia and $1.8 \%$ for cachara, the values were below the recommended for tanned leathers with salt chrome. Extractable substances in dichloromethane for salmon were the most referenced (14.5\%), while the rest were above the recommended amount, requiring adjustments in the formulation to obtain best quality leathers.

\section{INTRODUÇÃO}

Atualmente o Brasil produz aproximadamente 1,25 milhões de toneladas de pescado, sendo $38 \%$ cultivados. A atividade gera um PIB pesqueiro de $\mathrm{R} \$ 5$ bilhões, proporcionando 3,5 milhões de empregos diretos e indiretos. O potencial brasileiro é enorme e o país pode se tornar um dos maiores produtores mundiais de pescado (MPA, 2011). Grande parte da produção da piscicultura é comercializada na forma de filé, tendo as peles como resíduos da filetagem. Nesse processo, a pele de peixe, que representa em média 7,5\% do seu peso total, é considerada um subproduto que pode ser beneficiado por um processo de curtimento e transformado em couro (Franco, 2011).

Esse couro pode ser utilizado para diversas finalidades, tais como aplicação na indústria de confecção de vestuário, de calçados e de artefatos de decoração. Todavia, dependendo da finalidade há necessidade de avaliar a qualidade de resistência. Existem diversos fatores que influenciam na resistência do couro, tais como: a espécie de peixe, idade, peso, sentido da pele ou comprimento do peixe (transversal ou longitudinal) e o processo de curtimento. O processo de curtimento está altamente re- 
lacionado com os tipos e concentrações de produtos químicos utilizados (óleos, os diversos curtentes, ácidos e enzimas), o tempo de processo e a ação mecânica em cada etapa do processo (Souza et al., 2007). Quanto à espécie, existem diferenças acentuadas entre as peles das várias espécies de peixes, em função da estrutura histológica (disposição e orientação das fibras colágenas), as quais necessitam de técnicas diferenciadas de curtimento, seja em tempo ou quantidade de produtos, acréscimo de um ou outro produto químico, até mesmo a inclusão de mais etapas (Adeodato, 1998), tais como fazer várias vezes a etapa de desengraxe em relação ao maior teor de gordura natural presente na pele de determinadas espécies de peixes.

Estudos anteriores (Adeodato, 1998; Sacco, 1998) mostram que as peles de peixe, após o curtimento e na mesma espessura do couro bovino, apresentam alta resistência. Dessa forma, o aproveitamento dos subprodutos gerados pelo abate dos peixes, principalmente a pele, é uma excelente fonte de matériaprima para a confecção de vários produtos, desde que tenha sido processada com técnica adequada de curtimento.

A tilápia é a espécie que apresenta o maior número de trabalhos utilizando a sua pele para o curtimento. Diferentes técnicas de curtimento (Souza e Silva, 2005) e métodos de remoção da pele (Souza et al., 2006), testes físico-químicos (Godoy et al., 2010), testes mecânicos (Souza et al., 2007) uso de taninos vegetais e sintéticos (Vieira et al., 2008) já foram relatados na literatura. Por outro lado, ressalta-se que trabalhos utilizando a pele de cachara (Souza et al., 2014) e salmão (Franco et al., 2013) são escassos e ainda não há uma formulação definida para estas espécies.

Desta forma objetivou-se realizar análises físicoquímicas e mecânicas do couro de três espécies de peixes: tilápia do Nilo, cachara e salmão submetidos à mesma técnica de curtimento para avaliação da resistência desses couros.

\section{MATERIAL E MÉTODOS}

Foram utilizadas peles de tilápia do Nilo (Oreochromis niloticus), salmão (Salmo salar) e cachara (Pseudoplatystoma fasciatum), obtidas respectivamente de unidade de beneficiamento e peixarias na cidade de Rolândia - PR, Maringá - PR e Corumbá - MS. As peles foram acondicionadas em sacos plásticos e congeladas até a realização do processo de curtimento.

Para o curtimento, as peles foram descongeladas em temperatura ambiente e descarnadas (remoção de músculo presente na camada inferior da pele, hipoderme). As peles foram pesadas, para cálculo da quantidade de água e produtos químicos utilizados para cada espécie em cada fase do processo de curtimento (tabela I). A formulação foi baseada em trabalhos de Souza et al. (2003) e Souza (2004) com algumas adaptações na porcentagem dos produtos utilizados e no tempo, considerando a sensibilidade principalmente da pele de salmão, no processo de gelatinização e da cachara para evitar a despigmentação.

Utilizou-se fulões para o curtimento das peles de tilápia e salmão, enquanto que para as peles de cachara utilizou-se balde de 20 litros. Este procedimento foi necessário em função de práticas realizadas anteriormente em fulão que ocorreu a despigmentação das peles pela ação mecânica exercida pelo próprio equipamento.

Após curtimento das peles estas foram submetidas aos cortes dos corpos-de-prova para realizar os testes de resistência. Foram retirados quinze corposde-provas dos couros com uso de balacim (ABNT 11035, 1990), no sentido longitudinal (comprimento do corpo dos peixes para a determinação da resistência à tração, ao alongamento (ABNT 11041, 1997) e ao rasgamento progressivo (ABNT 11055, 1997). Em seguida foram determinadas as espessuras de cada amostra (ABNT 11062, 1997) com auxílio de espessímetro. Os corpos-de-prova dos couros foram encaminhados a um laboratório climatizado $\left(23^{\circ} \mathrm{C}\right.$ e $50 \%$ de umidade relativa do ar) por 24 horas, (ABNT 10455, 1988). Para realização destes testes de resistência, foi utilizado dinamômetro EMIC com velocidade de afastamento entre cargas de $100 \pm 20 \mathrm{~mm} \cdot \mathrm{min}^{-1}$.

Realizou-se as análises físico-químicas para a determinação do óxido de cromo $\left(\mathrm{Cr}_{2} \mathrm{O}_{3}\right)$ (ABNT 1105, 1999), substâncias extraíveis com diclorometano $\left(\mathrm{CH}_{2} \mathrm{Cl}_{2}\right)(\mathrm{ABNT} 11030,1997)$, pH e a cifra diferencial do pH de um extrato aquoso (ABNT 11057, 1999).

Os resultados dos testes físico-mecânicos foram submetidos à análise de variância e as médias foram comparadas pelo teste de Tukey com 95\% de confiabilidade.

\section{RESULTADOS E DISCUSSÃO}

As espessuras médias dos couros variaram de 0,68 a $0,86 \mathrm{~mm}$ para os corpos de prova (tabela II) entre as espécies. O couro da tilápia do Nilo $(22,17$ $\mathrm{N} / \mathrm{mm}^{2}$ ) apresentou maior resistência à tração quando comparado ao couro de salmão $\left(18,21 \mathrm{~N} / \mathrm{mm}^{2}\right)$ e foi estatisticamente semelhante ao cachara $(21,03$ $\mathrm{N} / \mathrm{mm}^{2}$ ). A espessura dos couros analisados por Almeida (1998) foi inferior $(0,41 \mathrm{~mm})$ ao relatado para couro de tilápia no presente trabalho, porém, foram semelhantes na tração $\left(22,16 \mathrm{~N} / \mathrm{mm}^{2}\right)$. De acordo com Souza e Silva (2005) e Viera et al. (2008) a espessura dos couros podem variar de acordo com a técnica de recurtimento aplicada (utilização de tanino vegetal, sintético a combinação de ambos ou sais de cromo), bem como o sentido dos cortes. Desta forma, a determinação da espessura do couro está diretamente relacionada com a sua resistência e consequentemente a aplicação deste material na indústria.

Quanto à elongação até a ruptura, não houve diferença entre a tilápia $(108,43 \%)$ e o salmão $(111 \%)$, sendo estes superiores ao cachara (58,93\%). Para a carga de ruptura, a tilápia do Nilo apresentou valor superior $(189,71 \mathrm{~N})$ às demais espécies. Os corpos- 
Tabela I. Formulação utilizada para as três espécies curtidas (Formulation used for the leather of the three species).

\begin{tabular}{|c|c|c|c|}
\hline Produto & Quantidade (\%) & Tempo (min.) & Observações \\
\hline \multicolumn{4}{|c|}{ Desengraxe - Processo realizado duas vezes } \\
\hline Tensoativo MK IV® & 0,5 & \multirow[t]{2}{*}{30} & Esgotar \\
\hline Água & 200 & & Lavar \\
\hline \multicolumn{4}{|l|}{ Caleiro 1} \\
\hline Dermaphel Plus $®$ & 8 & \multirow{5}{*}{60} & \multirow{5}{*}{$\begin{array}{l}\text { Esgotar } \\
\text { Lavar }\end{array}$} \\
\hline Bicarbonato de sódio & 2,5 & & \\
\hline Oxido de cálcio & 0,5 & & \\
\hline Tensoativo MK IV® & 0,5 & & \\
\hline Água & 200 & & \\
\hline \multicolumn{4}{|l|}{ Caleiro 2} \\
\hline Dermaphel Plus $®$ & 8 & \multirow{5}{*}{30} & \multirow{7}{*}{$\begin{array}{l}\text { Após } 60 \text { minutos as peles pernoitaram em banho } \\
\text { estático. } \\
\text { Esgotar } \\
\text { Lavar }\end{array}$} \\
\hline Bicarbonato de sódio & 2,5 & & \\
\hline Oxido de cálcio & 1 & & \\
\hline Tensoativo MK IV® & 0,5 & & \\
\hline Água & 50 & & \\
\hline Dermaphel Plus $®$ & 3 & 5 & \\
\hline Óxido de cálcio & 0,5 & 25 & \\
\hline \multicolumn{4}{|l|}{ Desencalagem 1} \\
\hline Sulfato de amônia & 2 & \multirow[t]{4}{*}{20} & \multirow[t]{4}{*}{$\begin{array}{l}\text { Esgotar } \\
\text { Lavar }\end{array}$} \\
\hline Dekalon® & 1 & & \\
\hline Tensoativo MK IV® & 0,5 & & \\
\hline Água & 100 & & \\
\hline \multicolumn{4}{|l|}{ Desencalagem 2} \\
\hline Dekalon® & 1 & & \multirow{3}{*}{$\begin{array}{l}\text { Esgotar } \\
\text { Lavar }\end{array}$} \\
\hline Tensoativo MK IV® & 0,5 & & \\
\hline Água & 100 & & \\
\hline \multicolumn{4}{|l|}{ Purga } \\
\hline Korapon NPA® & 0,3 & \multirow{3}{*}{30} & \multirow{3}{*}{$\begin{array}{l}\text { Esgotar } \\
\text { Lavar }\end{array}$} \\
\hline Tensoativo MK IV® & 0,5 & & \\
\hline Água & 100 & & \\
\hline \multicolumn{4}{|l|}{ Desengraxe } \\
\hline Dekalon $®$ & 2 & \multirow{3}{*}{20} & \multirow{3}{*}{$\begin{array}{l}\text { Esgotar } \\
\text { Lavar }\end{array}$} \\
\hline Tensoativo MK IV® & 0.5 & & \\
\hline Água & 100 & & \\
\hline \multicolumn{4}{|l|}{ Píquel } \\
\hline Cloreto de sódio & $90 \mathrm{~g} / \mathrm{kg}$ de pele & 10 & \\
\hline Ácido Fórmico & 1,5 & 10 & \\
\hline Ácido Fórmico & 1,5 & 20 & $\mathrm{VBC}=$ cor amarela, $\mathrm{pH} 3,0$ \\
\hline \multicolumn{4}{|l|}{ Curtimento } \\
\hline Chromosal B & 10 & 60 & Mesmo banho de píquel \\
\hline \multicolumn{4}{|l|}{ Basificação } \\
\hline Formiato de sódio & 1 & \multirow{2}{*}{15} & \\
\hline Bicarbonato de sódio & 0,5 & & \\
\hline Bicarbonato de sódio & 0,5 & 15 & \\
\hline Bicarbonato de sódio & 0,5 & 15 & \\
\hline Neutralização & & & \\
\hline Bicarbonato de sódio & 1 & & Após 60 minutos os couros pernoitaram em banho \\
\hline Tensoativo MK IV® & 0,5 & 60 & $\begin{array}{l}\text { estático. } \\
\text { Esgotar/Lavar }\end{array}$ \\
\hline
\end{tabular}

Archivos de zootecnia vol. 65, núm. 251, p. 351. 
Tabela I (continuação). Formulação utilizada para as três espécies curtidas (Formulation used for the leather of the three species).

\begin{tabular}{llll}
\hline Recurtimento e tingimento & & & \\
\hline Tamol® & 1 & 60 & $\begin{array}{l}\text { Esgotar } \\
\text { Lavar }\end{array}$ \\
Tanino vegetal (Weibull®) & 2 & & \\
Tanino sintético (Syntac F®) & 2 & & \\
Corante & 1,5 & 60 & Esgotar \\
\hline Engraxe & 7 & & Lavar \\
\hline Óleo (Superdema GW) & 100 & 30 & Esgota/lavar \\
Água & & 30 & \\
Fixação & 1 & & \\
Ácido fórmico & 7 & & \\
\hline
\end{tabular}

de-prova de cachara $(144,36 \mathrm{~N})$ e salmão $(128,71 \mathrm{~N})$ não apresentaram diferença significativa $(p>0,05)$ entre si. De acordo com Junqueira et al. (1983), o arranjo estrutural das fibras colágenas da derme permite que a pele possua grande resistência às diferentes forças de tração. Dependendo da espécie de peixe, a pele pode apresentar diferença na resistência em relação ao sentido ou à posição do couro, as metodologias de curtimento e quanto aos agentes curtentes utilizados no curtimento (Franco, 2011). Por essa razão, as peles de algumas espécies de peixes podem ser utilizadas na confecção de vestuários, pela sua maior resistência.

Segundo Souza et al. (2008), o couro de tilápia do Nilo no sentido longitudinal apresenta maior elasticidade, ou seja, o valor médio para o teste de alongamento sempre será maior. Os mesmos autores também relataram sobre a resistência do couro de pacu (Piaractus mesopotamicus), onde analisando o sentido transversal, os corpos de prova apresentaram valor médio de tração de $13,81 \mathrm{~N} / \mathrm{mm}^{2}$ e $76,98 \%$ de alongamento, enquanto no sentido longitudinal os valores médios foram significativamente inferiores $\left(5,93 \mathrm{~N} / \mathrm{mm}^{2}\right.$ e $52,20 \%$, respectivamente). Portanto, assim mostra que existe diferença na resistência dos couros de peixes em função da espécie e até mesmo na posição ou sentido do couro avaliado.

Souza et al. (2014) avaliaram a resistência de couros de cachara em função da adição de óleos $(8 \%$ a $16 \%$ ) na etapa de engraxe do processo de curtimen- to. Os autores relataram que o couro de cachara com espessura de 0,66 a 0,98 mm apresentaram resistência a tração que variou de $17,72 \mathrm{~N} / \mathrm{mm}^{2}$ a $29,15 \mathrm{~N} /$ $\mathrm{mm}^{2}$ e para elongação de $64,63 \%$ a $84,13 \%$. Os autores mencionados obtiveram maior maciez quando comparado ao obtido para a mesma espécie no presente trabalho, decorrente da maior elasticidade e a maior adição de óleo. Levando-se em consideração a porcentagem de $7 \%$ de óleo utilizado neste trabalho, os resultados para resistência a tração foi superior $\left(21,04 \mathrm{~N} / \mathrm{mm}^{2}\right)$ ao encontrado por Souza et al. (2014), apesar da menor elasticidade do couro de cachara $(58,93 \%)$ deste trabalho. Portanto, as características físico-mecânicas também podem ser melhoradas pela ação do engraxe, propiciando maior resistência à tração e elongação, como visto nos resultados mencionados anteriormente. Gutterres (2001) afirma que as substâncias engraxantes são, ao lado das de ação curtente, componentes principais incorporados à estrutura da pele em termos de massa durante a fabricação do couro.

Quanto à utilização dos couros de peixes para a confecção de vestuários, os de tilápia do Nilo e salmão podem ser utilizados, porém os couros de cachara apresentaram valor médio de alongamento inferior aos valores de referência para mamíferos de acordo com Hoinacki (1989), não sendo indicado para essa aplicação. Para a confecção de calçados, os couros das três espécies podem ser utilizados, pois segundo a Escola de Curtimento SENAI (Hoinacki, 1989), exige-se

Tabela II. Determinação dos testes de tração e alongamento dos couros de tilápia do Nilo, cachara e salmão, submetidos à mesma técnica de curtimento (Determination of tensile and elongation tests for Nile tilapia, cachara and salmon leather subjected to the same tanning technique).

\begin{tabular}{|c|c|c|c|c|}
\hline \multirow[t]{2}{*}{ Espécie } & \multicolumn{4}{|c|}{ Tração e alongamento } \\
\hline & $\begin{array}{l}\text { Espessura } \\
(\mathrm{mm})\end{array}$ & Carga de ruptura $(\mathrm{N})$ & Tração (N/mm²) & Elongação (\%) \\
\hline Tilápia do Nilo & $0,86^{a}$ & $189,71^{a}$ & $22,17^{a}$ & $108,43^{a}$ \\
\hline Cachara & $0,68^{b}$ & $144,36^{b}$ & $21,04^{\mathrm{ab}}$ & $58,93^{\mathrm{b}}$ \\
\hline Salmão & $0,71^{b}$ & $128,71^{\mathrm{b}}$ & $18,21^{b}$ & $111^{\mathrm{a}}$ \\
\hline Teste de F & $11,46^{\star *}$ & $9,73^{* *}$ & $3,25^{\star *}$ & $51,78^{* *}$ \\
\hline CV (\%) & 14,12 & 24,64 & 20,68 & 16,44 \\
\hline
\end{tabular}

a,b Valores seguidos de mesma letra, nas colunas, não diferem pelo teste Tukey a $5 \%$ de probabilidade. CV= coeficiente de variação.

**Significante $(p<0,05)$. 
Tabela III. Resultados dos testes de rasgamento progressivo dos couros de tilápia do Nilo, cachara e salmão submetidos à mesma técnica de curtimento (Results of progressive tearing tests for Nile tilapia, cachara and salmon leather subjected to the same tanning technique).

\begin{tabular}{|c|c|c|c|c|}
\hline \multirow{2}{*}{ Espécie } & \multicolumn{4}{|c|}{ Rasgamento progressivo } \\
\hline & Espessura (mm) & Rasgo (N/mm) & Força média (N) & Força máxima $(\mathrm{N})$ \\
\hline Tilápia do Nilo & $0,85^{a}$ & $79,17^{a}$ & 54,93 & 67,71 \\
\hline Cachara & $0,68^{b}$ & $82,36^{\mathrm{ab}}$ & 52,43 & 55,86 \\
\hline Salmão & $0,65^{b}$ & $105,69^{b}$ & 44,50 & 67,50 \\
\hline Teste de F & $12,78^{* *}$ & $3,47^{* *}$ & $1,58^{\text {ns }}$ & $1,45^{\text {ns }}$ \\
\hline CV (\%) & 15,65 & 33,92 & 32,01 & 33,09 \\
\hline
\end{tabular}

a,b Valores seguidos de mesma letra, nas colunas, não diferem pelo teste Tukey a $5 \%$ de probabilidade. CV= coeficiente de variação. **Significante $(p<0,05)$.

no mínimo resistência à tração de $17,65 \mathrm{~N} / \mathrm{mm}^{2}$, cujo valor foi obtido.

Nos testes de rasgamento progressivo, o couro de tilápia do Nilo apresentou-se significativamente $(\mathrm{p}<0,05)$ mais espesso que o couro de cachara e salmão $(0,854$ $\mathrm{mm}, 0,684$ e $0,65 \mathrm{~mm}$, respectivamente). Na determinação do rasgo do corpo de prova, o de tilápia $(79,19$ $\mathrm{N} / \mathrm{mm})$ diferiu-se do salmão $(105,69 \mathrm{~N} / \mathrm{mm})$ e o cachara $(82,36 \mathrm{~N} / \mathrm{mm})$ foi semelhante às duas espécies (tabela III).

A força média e máxima para o rasgamento dos couros não apresentou diferenças estatísticas $(p>0,05)$ entre as três espécies estudadas, com valores que variaram de 44,50 a 54,93 N para força média e 55,86 a 67,71 $\mathrm{N}$ para força máxima aplicada no teste.

Os resultados de rasgamento progressivo para tilápia foi superior ao descrito por Souza et al. (2006) e Godoy et al. (2010), estes autores encontraram valores de 27,91 (tilápia do Nilo) e 18,6 N/mm (tilápia vermelha) respectivamente, utilizando a técnica de curtimento com cromo e com corpos-de-prova retirados no sentido longitudinal. Souza et al. (2014) realizaram testes de resistência para couros de cachara que permaneceram em diferentes tempos de caleiro e encontraram valores inferiores para o rasgo $(56,61 \mathrm{~N} / \mathrm{mm})$ em peles que pernoitaram no caleiro, assim como o realizado neste estudo.

Analisando o couro de pacu (Piaractus mesopotamicus), Souza et al. (2008) obtiveram valor de rasgamento progressivo de $15,66 \mathrm{~N} / \mathrm{mm}$. Souza et al. (2003) relataram valores para piavuçu (Leporinus macrocephalus) de $8,45 \mathrm{~N} / \mathrm{mm}$, pacu prata (Mylossoma sp) de $36,51 \mathrm{~N} /$ $\mathrm{mm}$ e piraputanga (Brycon hilarii) de $8,16 \mathrm{~N} / \mathrm{mm}$ para o mesmo parâmetro. Todos estes couros analisados pelos autores relacionados anteriormente apresentaram menor resistência ao rasgamento progressivo ao comparado com o encontrado para salmão $(105,69 \mathrm{~N} / \mathrm{mm})$. Isto pode estar associado à técnica de curtimento, a espessura da pele dos animais e, sobretudo a estrutura histológica, ou seja, a direção e orientação das fibras colágenas. O valor mínimo estabelecido pela Basf (2005) para a resistência ao rasgo é de $35 \mathrm{~N} / \mathrm{mm}$ e os valores obtidos no presente estudo, para as três espécies estão acima do recomendado.

A quantidade de óxido de cromo está relacionada com a proporção deste curtente fixado às fibras colágenas. Desta forma, o valor de óxido de cromo no couro deve estar acima de 3,0\% (Hoinack, 1989), para que se obtenha bom resultado no teste de fervura ou retração e não ocorra a desnaturação do colágeno. Já, Basf (2005) afirma que o valor de óxido de cromo no couro deve estar acima de 2,5\%. No entanto, as quantidades de óxido de cromo encontrados neste trabalho são inferiores ao indicado na literatura para cachara $(1,8 \%)$ e tilápia $(2,0 \%)$; e no salmão o valor encontrado foi de 2,6\% (tabela IV), estando de acordo com o estabelecido por Basf (2005). Cabe ressaltar que toda a formulação, bem como o tempo em que as peles permaneceram em cada etapa foi baseado na pele do salmão, pelo fato do salmão ser um peixe de águas frias, a sua pele requer maior atenção no processo de curtimento, pois é uma pele extremamente delicada e há etapas no processo de curtimento que ocorre reação exotérmica (caleiro e píquel) tornando a pele mais sensível a desnaturação. Outra etapa que exige atenção é a purga, pois é utilizada uma enzima proteolítica, atuando diretamente no tecido dérmico. Esta enzima em excesso resultará na digestão da pele pela desnaturação das fibras colágenas e perda da estrutura dérmica ou em menor, mas não menos importante nível, o enfraquecimento do couro (Franco et al., 2013).

Tabela IV. Teor de óxido de cromo, graxa, pH e cifra diferencial das amostras de couro de tilápia do Nilo, cachara e salmão (Percentage of chromium oxide, fat, $\mathrm{pH}$ value and differential figure of Nile tilapia, cachara and salmon leather).

\begin{tabular}{|c|c|c|c|}
\hline \multirow{2}{*}{ Análise físico-química } & \multicolumn{3}{|c|}{ Espécie } \\
\hline & Tilápia do Nilo & Cachara & Salmão \\
\hline Determinação do óxido de cromo $\left(\mathrm{Cr}_{2} \mathrm{O}_{3}\right)(\%)$ & 2,0 & 1,8 & 2,6 \\
\hline Determinação de substâncias extraíveis em diclorometano (\%) & 28,7 & 22,6 & 14,7 \\
\hline Determinação do pH em extrato aquoso - base seca (\%) & 3,68 & 3,50 & 3,44 \\
\hline Determinação da cifra diferencial em extrato aquoso - base seca (\%) & 0,34 & 0,43 & 0,62 \\
\hline
\end{tabular}


A análise de substâncias extraíveis com diclorometano indica o conteúdo de óleos e graxas no couro. Em couros utilizados para vestuários, as substâncias extraíveis em diclorometano deveriam ser no máximo entre 16 a 18\% (Basf, 2005). Os resultados obtidos neste trabalho foram de 22,6, 28,7 e 14,7\% para tilápia, cachara e salmão respectivamente.

Grande quantidade de ácido no interior do couro pode ocasionar problemas, como o enfraquecimento com o tempo de armazenamento e uso. Os valores muito baixos de $\mathrm{pH}$ podem comprometer a fixação dos óleos utilizados no engraxe, resultando em baixos valores para as substâncias extraíveis em diclorometano, assim, os couros não apresentarão boa maciez e elasticidade. Segundo Hoinacki (1989) o valor mínimo de $\mathrm{pH}$ no couro deve ser de 3,5 e a cifra diferencial no máximo 0,7. A quantidade de ácido encontrados no presente trabalho variaram de 3,44 a 3,68 e o salmão foi a espécie que apresentou o $\mathrm{pH}$ abaixo do desejado. Para a cifra diferencial o valor máximo obtido foi de 0,62 .

Souza et al. (2006) encontraram para tilápias do Nilo valores dos testes físico-químicos superiores ao encontrado neste trabalho, para a mesma espécie, com exceção ao pH, cujo valor obtido pelos autores citados foi 2,9. Em cacharas, Souza et al. (2014) também obtiveram a quantidade de óxido de cromo e substancias extraíveis em diclorometano inferior ao recomentado.

Desta forma, considerando que a formulação utilizada para o curtimento das peles foi estabelecida levando em conta a sensibilidade da pele de salmão, os resultados da análise físico-química indicam que a tilápia e o cachara foram prejudicados quanto à quantidade de óxido de cromo, apesar da boa resistência observada; maior atenção nas fases inicias do processamento (remolho, caleiro, desencalagem, purga e píquel) poderiam contribuir com a melhor penetração e fixação do agente curtente no couro. Para o salmão, seriam necessários ajustes na quantidade de ácidos e no engraxe, para proporcionar maior maciez nos couros, assim como mais estudos para as três espécies afim de proporcionar couros de qualidade e resistência.

\section{CONCLUSÕES}

Os melhores resultados para os testes mecânicos foram os obtidos para tilápia e salmão. Quanto aos testes físico-químicos, o salmão apresentou os melhores resultados, considerando-se que a formulação foi baseada na sensibilidade da pele de salmão. Há a necessidade de ajustes na formulação para a obtenção de couros de melhor qualidade.

\section{AGRADECIMENTOS}

Ao Programa de Pós-Graduação em Zootecnia da Universidade Estadual de Maringá pelo apoio técnico e ao Conselho Nacional de Desenvolvimento Científico e Tecnológico (CNPq) pelo apoio financeiro.

\section{BIBLIOGRAFIA}

Adeodato, S. 1995. Peles exóticas e ecológicas. Glob Cien, 51: 56-60.
Almeida, R.R. 1998. A pele de peixe tem resistência e flexibilidade?. Revis Couro, 127: 49-53.

ABNT 10455 - Associação Brasileira de Normas Técnicas. 1988. NBR 10455: climatização de materiais usados na fabricação de calçados e correlatos. Rio de Janeiro. pp. 1-2.

ABNT 11030 - Associação Brasileira de Normas Técnicas. NBR. 1997. 1 1030: couro - determinação de substâncias extraíveis com diclorometano $\left(\mathrm{CH}_{2} \mathrm{Cl}_{2}\right)$. Rio de Janeiro. pp. 1-3.

ABNT 11035 - Associação Brasileira de Normas Técnicas. 1990. NBR 11035: corte de corpos-de-prova em couro. Rio de Janeiro. p. 1.

ABNT 11041 - Associação Brasileira de Normas Técnicas. 1997. NBR 11041 : couros - determinação da resistência à tração e alongamento. Rio de Janeiro. pp. 1-5.

ABNT 11055 - Associação Brasileira de Normas Técnicas. 1997. NBR 11055: couro - determinação da força de rasgamento progressivo. Rio de Janeiro. pp. 1-4.

ABNT 11062 - Associação Brasileira de Normas Técnicas. 1997. NBR 1 1062: determinação da espessura. Rio de Janeiro. p. 1.

ABNT 11054 - Associação Brasileira de Normas Técnicas. 1999. NBR 11054: couros determinação de óxido crômico $\left(\mathrm{Cr}_{2} \mathrm{O}_{3}\right)$. Rio de Janeiro. pp. 1-5.

ABNT 11057 - Associação Brasileira de Normas Técnicas. 1999. NBR 11057: couro - determinação do pH e da cifra diferencial. Rio de Janeiro. pp. 1-3.

BASF. 2005. Vademécum do curtidor. Ludwighafen. São Paulo.

Franco, M.L.R.S. 2011. Transformação da pele de peixe em couro. In: Ed. Gonçalves, A.A. Tecnologia do Pescado: Ciência, Tecnologia, Inovação e Legislação. Atheneu. São Paulo.

Franco, M.L.R.S.; Uchimura, C.M.; Prado, M.; Yajima, E.M.; Gasparino, E. e Silva, S.C.C. 2013. Qualidade da pele do salmão, Salmo solaris: teste de resistência e hidroxiprolina. Arq Cien Mar, 46: 90-95.

Godoy, L.C.; Gasparino, E.; Franco, M.L.R.S.; Franco, N.P. e Dourado, D.M. 2010. Testes físico-mecânicos e físico-químicos do couro da tilápia vermelha. Arq Bras Med Vet Zoo, 62: 475-480.

Gutterres, M. 2011. Distribuição, deposição e interação química de substâncias de engraxe no couro. Congresso da Federação Latino- Americana das Associações dos Qúmicos e Técnicos da Indústria do Couro. Federação Latino-Americana das Associações dos Químicos e Técnicos da Indústria do Couro. Salvador.

Hoinacki, E. 1989. Peles e couros; origens, defeitos, industrialização. In Peles e couros; origens, defeitos, industrialização. SENAI, São Paulo. Junqueira, L.C.U.; Joazeiro, P.P.; Montes, G.S.; Menezes, N. e Pereira Filho, M.E. 1983. É possível o aproveitamento industrial da pele dos peixes de couro. Tecnicouro, 5: 4-6.

MPA - Ministério da Pesca e Aquicultura. 2011. Boletim Estatístico da Pesca e Aquicultura. 2011.60 pp.

Sacco, D. 1998. Curtimento transforma pele de peixe em objetos da moda. Revis Unesp Rural, 12: 24-26.

Souza, M.L.R.; Cucatti, T.; Andrade, M.B.; Godoy, L.C.; Tozzi, M.A.C.; Cavallieri, R. F. e Silva, L.O. 2004. Diferentes técnicas de recurtimento em peles de tilápia-do-Nilo (Oreochromis niloticus): qualidade de resistência. Ensaios Cienc, 8: 195.

Souza, M.L.R.; Ganeco, L.N.; Nakaghi, L.S.O.; Faria, R.H.S.A.; Wagner, P.M.; Povh, J.A. e Ferreira, I.C. 2008. Histologia da pele do pacu (Piaractus mesopotamicus) e testes de resistência do couro. Acta Sci Anim Sci, 25: 37-44.

Souza, M.L.R.; Prado, M.; Fernandes, V.R.T.; Delbem, A.C.B.; Lara, J.A.F.; Bielawski, K.; Gasparino, E. e Vesco, A.P.D. 2014. Pele de surubim: morfologia e resistência do couro com adição de óleos no engraxe. Acta Tecnol, 9: 24-31.

Souza, M.L.R. e Silva, L.O. 2005. Efeito de técnicas de recurtimento sobre a resistência do couro da tilápia do Nilo (Oreochromis niloticus L.). Acta Sci Anim Sci, 27: 535-540.

Souza, M.L.R.; Valdez, M.D.C.A.; Hoch, A.L.V.; de Oliveira, K.F.; Matos, I.R. e Camin, A.M. 2007. Avaliação da resistência da pele de tilápia do Nilo (Oreochrmis niloticus) nos sentidos longitudinal, transversal 
e diagonal, depois de submetida ao curtimento com sais de cromo e recurtimento com agentes. Acta Sci Anim Sci, 28: 361-367.

Souza, M.L.R.D.; Casaca, J.D.M.; Nakaghi, S.O.; Franco, N.D.P.; Silva, L.O.D.; Dourado, D.M. e Viegas, E.M.M. 2006. Efeito da técnica de curtimento e do método utilizado para remoção da pele da tilápia-do-nilo sobre as características de resistência do couro. Rev Bras de Zootecn, 35: 1273-1280.
Souza, M.L.R; Dourado, D.M.; Machado, S.D.; Buccini, D.F.; Jardim, M.I.A.; Matias, R.; Correia, C. e Ferreira, I.C. 2003. Análise da pele de três espécies de peixes: histologia, morfometria e testes de resistência. Rev Bras Zootecn, 32: 1551-1559.

Vieira, A.M.; Kachba, Y.R.; Franco, M.L.R.S.; Oliveira, K.F.; Godoy, L.C. e Gasparino, E. 2008. Curtimento de peles de peixe com taninos vegetal e sintético. Acta Sci Anim Sci, 30: 359-363. 